\title{
DESENVOLVIMENTO DE MUDAS DE CATINGUEIRA EM DIFERENTES SUBSTRATOS E NÍVEIS DE LUMINOSIDADE
}

\author{
Cimille Gabrielle Cardoso Antunes ${ }^{*}$, Cíntia Luiza Mascarenhas de Souza ${ }^{2}$, Hugo Leonardo Rodrigues Gomes², \\ Jonaicon Vieira de Souza ${ }^{2}$, Natália dos Santos Barroso ${ }^{2}$, Renato Delmondez de Castro ${ }^{1}$, Claudinéia Regina Pelacani² \\ *Autora para correspondência: cimillebio@gmail.com
}

RESUMO: Poincianella pyramidalis Tul. (catingueira) é uma leguminosa endêmica do semiárido nordestino que se enquadra bem no contexto de espécies florestais brasileiras que ainda carecem de estudos quanto às condições ideais para seu desenvolvimento e estabelecimento em campo. Dessa forma, objetivou-se com este estudo avaliar o crescimento inicial de mudas da referida espécie submetidas a diferentes substratos e níveis de luminosidade. Plântulas de catingueira foram cultivadas em três condições de luminosidade (100, 50 e 30\%) e em três tipos de substratos: Terra vegetal (T), Areia lavada (A) e Terra vegetal + Areia lavada (T+A). Após seis meses, as mudas foram avaliadas quanto ao diâmetro do colo, altura, número de folhas e matéria seca. As mudas cultivadas a 30 e $50 \%$ de luminosidade, nos substratos terra vegetal e terra vegetal + areia apresentaram os melhores índices para cada uma das variáveis analisadas, sugerindo que durante a fase de crescimento inicial as mudas de catingueira apresentam requerimento nutricional elevado e certo sombreamento pode ser interessante para o desenvolvimento das plântulas nas condições de cultivo analisadas.

Palavras-chave: Caatinga, Poincianella pyramidalis, desenvolvimento, mudas.

\section{INITIAL GROWTH OF CATINGUEIRA SEEDLINGS IN DIFFERENT SUBSTRATES AND LUMINOSITY LEVELS}

\begin{abstract}
Poincianella pyramidalis Tul. (catingueira) is an endemic legume of the semiarid northeast that fits well in the context of Brazilian forest species that still lack of studies on the ideal conditions for their development and establishment in the field. Thus, the aim of this study was to evaluate the initial growth of seedlings of the species subjected to different substrates and light levels. Catingueira seedlings were grown in three light conditions (100, 50 and 30\%) and three substrates: Soil (So), Sand (Sa) and Soil + Sand $(S o+S a)$. After six months, the seedlings were evaluated for diameter, height, number of leaves and dry weight. The seedlings grown at 30 and 50\% light, in So and So + Sa showed the best results for all of the variables analyzed, suggesting that during the initial growth catingueira seedlings have higher nutritional requirement and certain degree of shading can be interesting for seedling development under the growth conditions examined.
\end{abstract}

Key-words: Caatinga, Poincianella pyramidalis, development, seedlings.

\section{INTRODUÇÃO}

Os constantes problemas ambientais no Brasil têm aumentado o interesse sobre o conhecimento de espécies florestais nativas, com vistas à preservação. Dessa forma, a maioria dos projetos que visam à recuperação de áreas degradadas e a exploração florestal depende da produção de suas mudas (CAMPOS; UCHIDA, 2002).

O tipo de substrato é um dos primeiros aspectos a ser pesquisado para se garantir a produção de mudas de boa qualidade (CAMPOS; UCHIDA, 2002). O substrato exerce uma influência marcante na arquitetura do sistema radicular e no estado nutricional das plantas, desempenhando papel importante na sobrevivência da muda no campo (HOFFMANN et al., 2001). Segundo Fonseca (2001), na escolha de um substrato deve-se observar as características físico-químicas, a espécie a ser

${ }^{1}$ Universidade Federal da Bahia - Salvador, BA, Brasil

${ }^{2}$ Universidade Estadual de Feira de Santana - Feira de Santana, BA, Brasil usada, além dos aspectos econômicos, como baixo custo e disponibilidade de acesso.

O estudo da luminosidade é também de fundamental importância para a avaliação do potencial das espécies arbóreas em programas de revegetação, já que a luz é um dos fatores críticos para o desenvolvimento de mudas em viveiro, fornecendo energia durante a fotossíntese (ALMEIDA et al., 2004; ATROCH et al., 2001). Dessa forma, modificações nos níveis de luminosidade nos quais uma espécie está submetida, podem condicionar diferentes respostas fisiológicas em suas características bioquímicas, anatômicas e de crescimento (ATROCH et al., 2001).

Frequentemente, as análises de crescimento são utilizadas para indicar vigor e grau de tolerância das espécies a diferentes condições ambientais, e os parâmetros morfológicos indicadores de qualidade mais utilizados nessas análises são: a altura, o diâmetro de

Cerne, Lavras, v. 20, n. 1, p. 55-60, jan./mar. 2014 
colo, o número de folhas e o acúmulo de massa seca que reflete o acúmulo de material resultante da fotossíntese líquida, sendo o aspecto fisiológico mais importante para a análise de crescimento (BENINCASA, 2003; CHAVES; PAIVA, 2004).

No presente trabalho, a espécie a ser estudada é a Poincianella pyramidalis (Tul.) L. P. Queiroz, uma leguminosa endêmica do semiárido nordestino que carece de estudos quanto às condições ideais para seu desenvolvimento e estabelecimento em campo. Vulgarmente conhecida como catingueira, destaca-se pelo seu potencial múltiplo (madeireiro, forrageiro e medicinal), sendo suas flores, folhas e cascas excelentes alternativas no tratamento de infecções respiratórias e estomacais (BRAGA, 1976). A espécie propaga-se por sementes, apresentando germinação rápida e adapta-se bem a vários tipos de substratos (MAIA, 2004; SALVAT et al., 2004).

Objetivou-se, com este trabalho, avaliar o desenvolvimento de mudas de catingueira submetidas a diferentes substratos e níveis de luminosidade, visando gerar informações úteis para utilização da espécie em programas de recuperação de áreas degradadas.

\section{MATERIAL E MÉTODOS}

\subsection{Material genético e local de coleta}

As sementes utilizadas foram coletadas a partir de espécimes de catingueira oriundas da Reserva Particular do Patrimônio Natural - Fazenda Morrinhos localizada

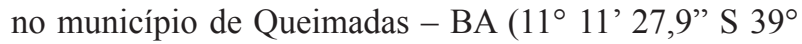
41 ' 26,6” W). Segundo a classificação de Köppen, o clima local é caracterizado como BSh (Clima semiárido quente) e a precipitação média anual se encontra entre 380 e $760 \mathrm{~mm}$, sendo que a taxa de evapotranspiração, média anual, geralmente supera a de precipitação (AMBIENTE BRASIL, 2008).

\subsection{Semeadura, tratamentos adotados e delineamento experimental}

Os experimentos foram conduzidos nos viveiros da Unidade Experimental Horto Florestal da Universidade Estadual de Feira de Santana-UEFS, Feira de Santana, BA, Brasil.

A semeadura foi realizada em sacos de polietileno com capacidade de $2,2 \mathrm{~kg}$, os quais continham os três tipos de substratos: terra vegetal (T), areia lavada (A) e terra vegetal + areia lavada $(1: 1 \mathrm{v} / \mathrm{v})(\mathrm{T}+\mathrm{A})$. A semeadura constou de duas sementes por saco, a uma profundidade de $2 \mathrm{~cm}$, desbastando-se uma das plântulas após emergência.
Em seguida, os recipientes com as sementes foram submetidos a três condições de luminosidade: $30 \%$ $\left(600 \mu \mathrm{mol} \mathrm{m} \mathrm{m}^{-2} \mathrm{~s}^{-1}\right), 50 \%\left(1000 \mu \mathrm{mol} \mathrm{m} \mathrm{m}^{-2} \mathrm{~s}^{-1}\right)$ e $100 \%$ $\left(2000 \mu \mathrm{mol} \mathrm{m}^{-2} \mathrm{~s}^{-1}\right)$.

Foram utilizados telados de sombrite para a obtenção dos níveis de 30 e $50 \%$ de luminosidade e para a condição de $100 \%$ de luminosidade as mudas foram colocadas diretamente sob o sol. A medição da intensidade luminosa de cada ambiente foi feita com o auxílio do IRGA - Portable Open System Infrared Gas Analyzer (LCi, ADC BioScientific Ltd., England).

Os tratos culturais adotados foram irrigações diárias (2 regas/dia); 1 aplicação foliar preventiva de inseticida (Dimy Pronto - Tetrametrina 0,5\% p/v) para o controle de pulgões e retirada semanal das plantas consideradas invasoras, a fim de evitar competição por luz e nutrientes. Utilizou-se o delineamento experimental inteiramente casualizado, em esquema fatorial $3 \times 3$ (três níveis de luminosidade $\mathrm{x}$ três tipos de substratos), com cinco repetições de uma planta por substrato e nível de luminosidade.

\subsection{Análise de crescimento}

Após seis meses da semeadura (período de dezembro de 2006 a junho de 2007), foram feitas as seguintes análises (BENINCASA, 2003; CLEMENT; BOVI, 2000):

1- Altura das plantas $(\mathrm{cm})$ : Utilizou-se régua milimetrada, medindo-se desde a superfície do solo, até o ápice da maior folha esticada na posição vertical.

2- Diâmetro de colo das mudas (mm): Foi medido na altura da superfície do solo com auxílio de um paquímetro digital (Cosa 111-101EB).

3- Número de folhas: Foram efetuadas contagens das folhas completamente expandidas de cada muda, em cada ambiente de luz e substrato testados.

4- Massa seca: Foi realizada a análise destrutiva das mudas, separando-se parte radicular da parte aérea. A porção radicular foi lavada em água corrente para a retirada do substrato. Em seguida, acondicionou-se parte aérea e radicular das mudas separadamente, em sacos de papel kraft para a secagem do material em estufa de ventilação forçada a $60^{\circ} \mathrm{C}$ até peso constante. A massa seca do material foi determinada em balança analítica.

\subsection{Análise estatística}

Os dados obtidos foram avaliados quanto à homocedasticidade e normalidade, de modo que aqueles que não atenderam aos pressupostos dos testes 
paramétricos sofreram transformação radical $\left(\mathrm{X}^{0,5}\right)$ (SNEDECOR; COCHRAN, 1989). Em seguida, tais dados foram submetidos à análise de variância e as médias foram comparadas pelo Teste Tukey a 5\% de probabilidade, por meio do programa estatístico SISVAR (FERREIRA, 1999).

\section{RESULTADOS E DISCUSSÃO}

De modo geral, o desenvolvimento das mudas de catingueira foi especialmente influenciado pelo substrato e pelo nível de luminosidade de maneira independente. A interação entre as fontes de variação substrato e luminosidade não foi significativa.

Os substratos T e T+A e as condições de 30 e $50 \%$ de luminosidade foram os tratamentos que propiciaram maior incremento em diâmetro de colo das mudas de catingueira, não diferindo estatisticamente entre si pelo teste Tukey $(0,05)$ (Tabela 1$)$.

Tabela 1 - Valores médios de diâmetro de colo ( $\mathrm{mm})$, altura $(\mathrm{cm})$, número de folhas, massa seca da parte aérea $(\mathrm{g})$ e massa seca da parte radicular (g) de mudas de catingueira (Poincianella pyramidalis Tul.) cultivadas em diferentes substratos e níveis de luminosidade com seis meses de idade. Feira de Santana, Ba, Brasil.

Table 1 - Average values of root-collar diameter $(\mathrm{mm})$, height (cm), number of leaves, dry matter of shoot $(\mathrm{g})$ and dry matter of root of catingueira seedlings (Poincianella pyramidalis Tul.) six months aged cultivated under different substrates and light regimes, Feira de Santana, BA, Brasil.

\begin{tabular}{lcccccc}
\hline \multirow{2}{*}{ Parâmetro } & \multicolumn{3}{c}{ Luminosidade } & \multicolumn{3}{c}{ Substrato } \\
\cline { 2 - 7 } & $100 \%$ & $50 \%$ & $30 \%$ & Terra & Areia & $\mathrm{T}+\mathrm{A}$ \\
\hline Diâmetro de colo & $4,61 \mathrm{~b}$ & $5,89 \mathrm{a}$ & $5,69 \mathrm{a}$ & $6,45 \mathrm{~A}$ & $4,16 \mathrm{~B}$ & $5,59 \mathrm{~A}$ \\
Altura & $24,5 \mathrm{~b}$ & $32,8 \mathrm{ab}$ & $40,6 \mathrm{a}$ & $46,1 \mathrm{~A}$ & $16,5 \mathrm{~B}$ & $34,3 \mathrm{~A}$ \\
$\mathrm{~N}$ folhas & $8,2 \mathrm{~b}$ & $12,2 \mathrm{a}$ & $14,5 \mathrm{a}$ & $13,5 \mathrm{~A}$ & $9,2 \mathrm{~B}$ & $12,1 \mathrm{~A}$ \\
Massa seca PA & $2,3 \mathrm{~b}$ & $5,1 \mathrm{a}$ & $3,8 \mathrm{a}$ & $7,9 \mathrm{~A}$ & $0,9 \mathrm{C}$ & $2,5 \mathrm{~B}$ \\
Massa seca Raiz & $1,7 \mathrm{~b}$ & $3,1 \mathrm{a}$ & $2,3 \mathrm{ab}$ & $4,0 \mathrm{~A}$ & $1,3 \mathrm{~B}$ & $1,8 \mathrm{~B}$ \\
\hline *Médias seguidas pela mesma letra minúscula (luminosidade)ou \\
$\begin{array}{l}\text { maiúscula (substrato) não diferem entre si pelo Teste Tukey a 5\% } \\
\text { de probabilidade. N Folhas = número de folhas; PA= parte aérea. }\end{array}$
\end{tabular}

Entre os parâmetros morfológicos que indicam a boa qualidade de uma muda, o diâmetro do colo é um dos mais importantes, pois o mesmo pode ser considerado como um critério para o plantio da muda em campo e, ao mesmo tempo, um bom índice relacionado à sobrevivência da muda, estando diretamente relacionado ao substrato utilizado (SCALON et al., 2002). As mudas cultivadas nos substratos $\mathrm{T}$ e $\mathrm{T}+\mathrm{A}$ (Tabela 1 ), foram as que apresentaram maior incremento em diâmetro, de modo que as características físicas desses substratos possam ter favorecido o desenvolvimento das mudas (CHARLO et al., 2006; CUNHA et al., 2006).

A necessidade de sombreamento para incremento em diâmetro de colo das mudas (Tabela 1), reflete uma estratégia da catingueira em requerer condições pouco estressantes de temperatura e de luz para estimular seu desenvolvimento inicial. Fonseca et al. (2006) também encontraram resultados semelhantes em plantas de Pseudopiptadenia psilostachya cultivadas sob 30 e 50\% de sombreamento. Alguns autores, no entanto, relatam maior investimento em diâmetro, em plantas submetidas a maiores intensidades luminosas como em Schizolobium parahyba (FERREIRA et al., 1977) e Erythrina speciosa (ENGEL, 1989) sob 100\% de luminosidade.

No que diz respeito à variável altura, as mudas de catingueira cultivadas no substrato $\mathrm{T}$ e $\mathrm{T}+\mathrm{A}$ foram as que apresentaram maior crescimento, sugerindo, assim, maior requerimento nutricional da espécie para alongamento inicial do eixo principal. O nível de $30 \%$ de luminosidade estimulou maior crescimento em altura das mudas (Tabela 1).

De acordo com Minami (1995) e Nicoloso et al. (2000), características em substratos como boa porosidade e densidade são fundamentais para favorecer o desenvolvimento das plântulas em formação. O menor crescimento em altura das mudas de catingueira no ambiente de $100 \%$ de luminosidade sugere que a intensidade luminosa mais elevada (2000 $\left.\mu \mathrm{mol} \mathrm{m} \mathrm{m}^{-2} \mathrm{~s}^{-1}\right)$, possa ter desencadeado algum tipo de condição estressante, haja vista que sob o sol pleno a taxa evapotranspiratória e respiratória das mudas é muito intensa, reduzindo o alongamento caulinar (BARBER; ANDERSON, 1992). Resultados semelhantes com relação à altura foram encontrados por Atroch et al. (2001) estudando o crescimento inicial de mudas de Bauhinia fortificata sob 50 e $70 \%$ de luminosidade.

Seguindo a mesma tendência das outras variáveis analisadas, as plantas crescidas em $\mathrm{T}$ e $\mathrm{T}+\mathrm{A}$ lançaram mais folhas. 30 e $50 \%$ de luminosidade também ofereceram as melhores condições de cultivo (Tabela 1). Apesar dessa característica não ser uma das mais indicadas para representar o crescimento das plantas quando submetidas a diferentes níveis de luz (já que as plantas durante seu ciclo de vida perdem e lançam folhas continuamente),

Cerne, Lavras, v. 20, n. 1, p. 55-60, jan./mar. 2014 
tal variável em conjunto com as demais, pode fornecer informações relevantes quanto ao comportamento das mesmas diante de variações do ambiente (BENINCASA, 2003; NODARI et al., 1999).

Resultados semelhantes foram encontrados por Paiva et al. (2003), estudando o crescimento inicial de mudas de Cassia grandis sob 50 e 100\% de luminosidade e por Câmara e Endres (2008) em sabiá (Mimosa caesalpiniifolia) sob 50\% de sombreamento.

No que diz respeito ao acúmulo de massa seca (parte aérea e parte radicular) das mudas de catingueira em função do substrato, pode-se perceber que o substrato $\mathrm{T}$ proporcionou maior incremento de material estrutural. Os níveis de 30 e $50 \%$ de luminosidade foram os que propiciaram os melhores resultados em ambos os casos, afastando a hipótese de estiolamento para o melhor desenvolvimento em altura das mudas submetidas às mesmas condições de luz (Tabela 1).

A resposta da catingueira à luz para acúmulo de biomassa mostrou-se diferencial, em virtude do perfil ecológico tipicamente pioneiro da espécie dentro do bioma Caatinga (MELO et al., 2004), demonstrando ampla plasticidade fenotípica da catingueira quando esta foi submetida à atenuação da irradiância. Muitas das características de alocação de recursos da planta variam com o nível de irradiância com o qual as espécies são aclimatadas ou ecologicamente restritas (GIVINISH, 1988). Plantas crescendo em ambientes mais sombreados adaptam-se à redução das taxas de respiração e alcançam mais rapidamente o ponto de compensação luminoso, compensando a diminuição do ganho de carbono nessa condição (LARCHER, 2003).

\section{CONCLUSÕES}

Os resultados sugerem que a catingueira se desenvolve bem nos substratos terra vegetal e terra vegetal + areia e que o sombreamento pode favorecer o crescimento. Espécies que apresentam plasticidade em caracteres ligados a sobrevivência, apresentam vantagens adaptativas em ambientes instáveis ou heterogêneos, resultando no aumento da tolerância ambiental.

\section{REFERÊNCIAS}

ALMEIDA, L. P.; ALVARENGA, A. A.; CASTRO, E. M.; ZANELA, S. M.; VIEIRA, C. V. Crescimento inicial de plantas de Cryptocaria aschersoniana Mez. submetidas a níveis de radiação solar. Ciência Rural, Santa Maria, v. 34, p. 83-88, 2004.

Cerne, Lavras, v. 20, n. 1, p. 55-60 jan./mar. 2014
AMBIENTE BRASIL. Classificação climática de Köppen-

Geiger. Disponível em: <http://www.ambientebrasil.com.br $>$. Acesso em: 30 jun. 2008.

ATROCH, E. M. A. C.; SOARES, A. M.; ALVARENGA, A. A.; CASTRO, E. M. Crescimento, teor de clorofilas, distribuição de biomassa e características anatômicas de plantas jovens de Bauhinia forticata LINK submetidas à diferentes condições de sombreamento. Ciência e Agrotecnologia, Lavras, v. 25, p. 853-862, 2001.

BARBER, J.; ANDERSON, B. Too much of a good thing: light can be bad for photosynthesis. Trends in Biochemical Sciences, Amsterdam, v. 17, p. 61-66, 1992.

BENINCASA, M. M. P. Análise de crescimento de plantas: noções básicas. 2. ed. Jaboticabal: FUNEP, 2003.

BRAGA, R. Plantas do nordeste: especialmente do Ceará. Natal: Fundação Guimarães Duque, 1976.

CÂMARA, C. A.; ENDRES, L. Desenvolvimento de mudas de duas espécies arbóreas: Mimosa caesalpiniifolia Benth. e Sterculia foetida L. sob diferentes níveis de sombreamento em viveiro. Floresta, Curitiba, v. 38, p. 43-51, 2008.

CAMPOS, M. A. A.; UCHIDA, T. Influência do sombreamento no crescimento de mudas de três espécies amazônicas. Pesquisa Agropecuária Brasileira, Brasília, v. 37, n. 3, p. 281-288, 2002.

CHARLO, H. C. O.; MORO, F. V.; SILVA, V. L.; SILVA, B. M. S.; BIANCO, S.; MORO, J. R. Aspectos morfológicos, germinação e desenvolvimento inicial de plântulas de Archontophoenix alexandre (F. Mueller) H. Wendl. E Drude (Arecaceae) em diferentes substratos. Revista Árvore, Viçosa, v. 30, n. 6, p. 933-940, nov./dez. 2006.

CHAVES, A. S.; PAIVA, H. N. Influência de diferentes períodos de sombreamento sobre a qualidade de mudas de fedegoso (Senna macranthera (Collad) Irwin et Barn). Scientia Forestalis, Piracicaba, v. 65, p. 22-29, 2004.

CLEMENT, C. R.; BOVI, M. L. A. Padronização de medidas de crescimento e produção de experimentos com pupunheira para palmito. Acta Amazônica, Manaus, v. 30, n. 3, p. 349$362,2000$. 
CUNHA, A. M.; CUNHA, G. M.; SARMENTO, R. A.; CUNHA, G. M.; AMARAL, J. F. T. Efeito de diferentes substratos sobre o desenvolvimento de mudas de Acacia $\mathrm{sp}$. Revista Árvore, Viçosa, v. 30, n. 2, p. 207-214, mar./abr. 2006.

ENGEL, V. L. Influência do sombreamento sobre o crescimento de mudas de essências nativas, concentração de clorofila nas folhas e aspectos de anatomia. 1989. 202 p. Dissertação (Mestrado em Ciências Florestais) - Escola Superior de Agricultura "Luiz de Queiroz", Piracicaba, 1989.

FERREIRA, D. F. SISVAR 4.3 - sistema de análises estatísticas. Lavras: UFLA, 1999.

FERREIRA, M. G. M.; CÂNDIDO, J. F.; CANO, M. A. O.; CONDE, A. R. Efeito do sombreamento na produção de mudas de quatro espécies florestais nativas. Revista Árvore, Viçosa, v. 1, n. 2, p. 121-134, 1977.

FONSECA, M. G.; LEÃO, N. V. M.; SANTOS, F. A. M. Germinação de sementes e crescimento inicial de plântulas de Pseudopiptadenia psilostachya (DC.) G.P.Lewis \& M.P.Lima (Leguminosae) em diferentes ambientes de luz. Revista Árvore, Viçosa, v. 30, n. 6, p. 885-891, nov./dez. 2006.

FONSECA, T. G. Produção de mudas de hortaliças em substratos de diferentes composições com adição de $\mathrm{CO}_{2}$ na água de irrigação. 2001. 72 p. Dissertação (Mestrado em Agronomia) - Escola Superior de Agricultura "Luiz de Queiroz”, Piracicaba, 2001.

GIVINISH, T. J. Adaptation to sun and shade a whole-plant perspectives: ecology of photosynthesis in sun and shade. Melbourne: CSINO, 1988.

HOFFMANN, A.; PASQUAL, M.; CHALFUN, N. N. J.; FRÁGUAS, C. B. Efeito de substratos na aclimatização de plantas micropropagadas do porta-enxerto de macieira 'Marubakaido'. Ciência e Agrotecnologia, Lavras, v. 25, n. 2 , p. 462-467, mar./abr. 2001.

LARCHER, W. Physiological plant ecology. New York: Springer-Verlag, 2003.

MAIA, G. N. Caatinga: árvores, arbustos e suas utilidades. São Paulo: D\&Z Computação Gráfica, 2004.
MELO, F. P. L.; AGUIAR NETO, A. V.; SIMABUKURO, E. A.; TABARELLI, M. Recrutamento e estabelecimento de plântulas. In: FERREIRA, A. G.; BORGHETTI, F. (Ed.). Germinação: do básico ao aplicado. São Paulo: Artmed, 2004. p. 237-250.

MINAMI, K. Produção de mudas em recipientes. São Paulo: T. A. Queiroz, 1995.

NICOLOSO, F. T.; FORTUNATO, R. P.; ZANCHETTI, F.; CASSOL, L. F.; EISINGER, S. M. Recipientes e substratos na produção de mudas de Maytenus ilicifolia e Apuleia leiocarpa. Ciência Rural, Santa Maria, v. 30, n. 6, p. 987-992, nov./dez. 2000.

NODARI, R. O.; REIS, M. S.; FANTINI, A. C.; MANTOVANI, A.; RUSCHEL, A.; WELTER, L. J. Crescimento de mudas de palmiteiro (Euterpe edulis Mart.) em diferentes condições de sombreamento e densidade. Revista Árvore, Viçosa, v. 23, n. 3, p. 285-292, maio/jun. 1999.

PAIVA, L. C.; GUIMARÃES, R. J.; SOUZA, C. A. S. Influência de diferentes níveis de sombreamento sobre o crescimento de mudas de cafeeiro (Coffea arabica). Ciência e Agrotecnologia, Lavras, v. 27, n. 1, p. 134-140, jan./fev. 2003.

SALVAT, A.; ANTONACCI, L.; FORTUNATO, R. H.; SUAREZ, E. Y.; GODOY, H. M. Antimicrobial activity in methanolic extracts of several plant species from northern Argentina. Phytomedicine, Jena, v. 11, p. 230-234, 2004.

SCALON, S. P. Q.; MUSSURY, R. M.; RIGONI, M. R.; VERALDO, F. Crescimento inicial de mudas de espécies florestais nativas sob diferentes níveis de sombreamento.

Revista Árvore, Viçosa, v. 26, n. 1, p. 1-5, jan./fev. 2002.

SNEDECOR, G. W.; COCHRAN, W. G. Statistical methods. Ames: Iowa State University, 1989.

Recebido: 16 de maio de 2011; aceito: 01 de julho de 2013.

Cerne, Lavras, v. 20, n. 1, p. 55-60, jan./mar. 2014 
